\title{
REVISTA PERSPECTIVA EDUCACIONAL: CRECIMIENTO E IMPACTO INTERNACIONAL
}

EDUCATIONAL PERSPECTIVE JOURNAL:

GROWTH AND INTERNATIONAL IMPACT

\section{REVISTA PERSPECTIVA EDUCACIONAL:} CRESCIMENTO E IMPACTO INTERNACIONAL

\section{RESUMEN}

María Verónica Leiva ${ }^{1}$ Natalia Paulina Hernández ${ }^{2}$

Aunque no existe un consenso único para definir la internacionalización de una revista se utiliza comúnmente como un sinónimo de calidad, ya que para alcanzar el grado de revista internacional es necesario haber superado numerosos obstáculos. En este artículo se dan a conocer algunas concepciones actuales acerca de la internacionalización y cuáles son las acciones que Perspectiva Educacional ha desarrollado para alcanzar el grado de revista internacional. Asimismo, se reflexiona acerca de los principales desafíos que debe superar para el logro pleno de ésta.

PALABRAS-CLAVE: Internacionalización. Revista Científica. Perspectiva Educacional. Conocimiento Científico.

\begin{abstract}
Although there is no single consensus to define the internationalization of a journal, this concept is commonly used as a synonym for quality, since to reach the degree of the international journal it is necessary to have overcome many obstacles. In this article, we present some current conceptions about internationalization and what are the actions that Perspectiva Educacional has developed to reach the degree of the international magazine. It also reflects on the main challenges that must be overcome for the full achievement of this.
\end{abstract}

KEYWORDS: Internationalization. Scientific Journal. Educational Perspective. Scientific Knowledge.

\section{RESUMO}

Ainda que não exista um consenso único para definir a internacionalização de uma revista, frequentemente esta é utilizada como um sinônimo de qualidade, já que para alcançar o grau de revista internacional é necessário ter superado inúmeros obstáculos. Neste artigo se apresentam algumas concepções atuais acerca da internacionalização e quais são as ações que a Perspectiva Educacional tem desenvolvido para alcançar o grau de revista internacional. Também se reflete acerca dos principais desafios que precisam ser superados para o seu pleno alcance.

PAlaVRAS-CHAVE: Internacionalização. Revista Científica; Perspectiva Educacional; Conhecimento Científico.

\footnotetext{
${ }^{1}$ Pontificia Universidad Católica de Valparaíso. E-mail: verleiva@gmail.com

${ }^{2}$ Pontificia Universidad Católica de Valparaíso . E-mail: natalia.hr@gmail.com Submetido em: 09/10/2016 - Aceito em: 11/11/2016

\begin{tabular}{l|l|l|l|l|l|l}
\hline (C) Rev. Inter. Educ. Sup. & Campinas, SP & v.2 & n.3 & p.480-500 & set./dez. 2016 & ISSN 2446-9424 \\
\hline
\end{tabular}
}




\section{Introducción}

En la actualidad existe un avance exponencial de la investigación y del conocimiento científico, y a la vez, la necesidad de exponer y discutir ideas innovadoras sobre las distintas áreas disciplinares. Las revistas científicas vienen a dar respuesta a estos requerimientos, de tal forma de generar discusión abierta y libre de los hallazgos experimentales, las hipótesis y las teorías, no sólo de las Ciencias Experimentales, sino también de las Sociales y las Humanidades (DELGADO, RUIZ; JIMÉNEZ, 2006).

En este sentido las revistas científicas también han sufrido cambios importantes en su proceso de edición que pueden explicarse con su búsqueda de internacionalización y difusión del conocimiento, pasando de formato impreso a un formato digital que permite mayor visibilidad, expansión del conocimiento y cooperación internacional. Al respecto Díaz y Ortiz (2014) señalan que la información se encuentra accesible y favorece la comunicación entre comunidades científicas. Esto implica uno de los avances más importantes para las revistas académicas ya que permite el libre acceso en la web, y ser consultada a nivel internacional 'por miles de personas a la vez, marcando una importante diferencia con el formato impreso.

Por otra parte, la necesidad de exponer y discutir ideas innovadoras genera desafíos a las revistas científicas que buscan su internacionalización.

Expandir la red internacional implica mejorar la calidad de los manuscritos seleccionados, conseguir un mayor flujo de manuscritos especializados de corriente principal, o de cobertura editorial geográfica más global, o de temas emergentes que incluyan autores con redes científicas internacionales" (ALOGERO et al., 2013, p. 368).

El presente artículo, pretende dar a conocer como se genera la internacionalización a partir de revistas científicas, específicamente desde la revista Perspectiva Educacional. Así, tras una definición del concepto de internacionalización, de Revista internacional, y de la revisión de criterios que otorgan calidad a una revista, se continúa con el análisis de las acciones que ha realizado Perspectiva Educacional con la intención de alcanzar la internacionalización.

\section{El Concepto de Internacionalización}

Aunque es un hecho evidente que la cuestión de la internacionalización de la educación superior está más presente que nunca, como se percibe claramente en la visibilidad de documentos nacionales internacionales y declaraciones de intenciones y estrategias institucionales (DE WIT, 2011), el fenómeno hunde sus raíces en la historia de la universidad, cuya universalización forma parte de su propia esencia, compartiendo además raíz gramatical (Knight, 2008 y 2010). 
La universidad siempre ha tenido cierto grado de internacionalización toda vez que, por la naturaleza de su actividad, formación superior, investigación y ascendencia social, se ha visto influenciada por la movilidad de su profesorado, así como por la divulgación de sus contribuciones. Por tanto, hay una primera acepción de la internacionalización que está ligada a la divulgación del conocimiento y redes de relación que van más allá de las fronteras locales y nacionales, generando así algún tipo de influencia que afecta a la comunidad internacional.

Algunos fenómenos de la realidad más reciente, caso de la globalización, el incremento masivo del número de universidades, de estudiantes universitarios, el avance del conocimiento, la imposición del inglés como lengua prioritaria de divulgación científica, han venido a contribuir a la magnificación del hecho de la internacionalización. Si a todo esto añadimos la incorporación de las Nuevas Tecnologías de la Información y Comunicación como vehículo de difusión y comunicación, este fenómeno de la internacionalización no ha hecho más que comenzar (ALTBACH, REISBERG; RUMBLEY, 2009).

En este sentido, la internacionalización puede ser entendida como una forma de colaboración internacional en el contexto de la educación superior, cuyos frutos más visibles serían las producciones científicas, el intercambio de estudiantes y de profesorado, así como el desarrollo de investigación compartida.

Sin embargo, a esta visión más positiva de la internacionalización, también se superpone en la actualidad otra no tan plausible, toda vez que la disponibilidad económica o la radicación en países más desarrollados de numerosas universidades, conlleva una capitalización en el monopolio de sistemas de investigación, autoría científica, control de redes y bases de datos o recursos de investigación que modifican o alteran esa concepción originaria de la internacionalización más cooperativa para adentrarse en un mundo de competitividad.

De este modo, hay que incorporar a esta visión de la universalización como cooperación otra visión, cada vez más generalizada, ligada al comercio de productos universitarios, ya se trate de docencia como de investigación y recursos de la información. Quizás, el fenómeno que más crecimiento esté experimentando en estos momentos sea el que tiene que ver con la venta de formación en base a una internacionalización de los planes de estudio y de los procesos formativos, que facilitan que estudiantes de todo el mundo puedan seguir estudios de determinados centros sin moverse de su casa, estableciéndose como una alternativa a la tradicional movilidad estudiantil, situación que puede generar más una situación de competencia que de cooperación entre las universidades.

Con todo, no es posible establecer un concepto unívoco de la internacionalización toda vez que las políticas nacionales, así como la naturaleza de cada una de las propias universidades, modulan el alcance de tal concepto de internacionalización, que pueden quedar más situadas en un contexto de colaboración-cooperación con vínculos de

\begin{tabular}{l|l|l|l|l|l|l}
\hline (C) Rev. Inter. Educ. Sup. & Campinas, SP & v.2 & n.3 & p.480-500 & set./dez. 2016 & ISSN 2446-9424
\end{tabular}


intercambios orientados a la potencialización de su propia calidad, siendo así que la internacionalización se establece como valor añadido, mientras que otras universidades puede situarse más en un escenario de mercado con claras políticas de competitividad para la captación de recursos, estudiantes, etc.

No obstante, entre los indicadores de internacionalización se hallan, sin duda, la divulgación o difusión del conocimiento, que puede realizarse mediante sistemas de edición en papel o digitales o la movilidad de las personas. Los repositorios de publicaciones, bases de datos y las revistas científicas son, claramente, potenciales instrumentos de internacionalización

\section{El Concepto de Revista Internacional}

Una revista científica es el instrumento más utilizado a la hora de comunicar los hallazgos científicos y resultados de la investigación, toda vez que, a su inmediatez, da la posibilidad de disponer de una frecuencia bastante elevada, de uno a tres o cuatro números anuales, se facilita su distribución, tanto si es en papel como si lo es de forma electrónica, formula ésta más versátil, ágil y de mayor grado de alcance en la distribución.

Una de las tareas propias de los investigadores, cuando consideran que su trabajo es pertinente, es la de ejercer influencia a través de su propio conocimiento en los demás investigadores de su ámbito.

Esta posibilidad de influjo es ejercida por medio de grupos o redes de mayor o menor grado de influencia en una mayor o menor amplitud del ámbito geográfico, llevándonos a visualizarlo en proyecciones de mayor o menor influencia según las posiciones contextuales de macrocontexto (internacional), mesocontexto (nacional) o microcontexto (más local o regional) con relación a cada ámbito o especialidad científica (DELGADO, RUÍZ-PÉREZ; JIMÉNEZ-CONTRERAS, 2016).

Así, por tanto, las revistas científicas vienen a justificarse por la oportunidad que ofrecen de intercambio en la producción del conocimiento, dando ocasión a la discusión abierta y libre de los hallazgos experimentales, las hipótesis y las teorías de las Ciencias Experimentales y de la Salud, de las Ciencias Sociales y de las Humanidades.

Adentrase en el concepto de revista Internacional, requiere analizar algunas de las variables que definen a la propia revista con relación a su proyección transfronteriza y que podría ser definida por el conjunto de aquellas variables que determinan que esa revista pueda definirse como internacional.

La primera variable es el establecimiento de una plataforma tecnológicamente avanzada para la edición y publicación de revistas, paso necesario y esencial para aproximar las revistas al estado del arte y fortalecer la competitividad internacional (Packer, 2014).

\begin{tabular}{l|l|l|l|l|l|l}
\hline (c) Rev. Inter. Educ. Sup. & Campinas, SP & v.2 & n.3 & p.480-500 & set./dez. 2016 & ISSN 2446-9424
\end{tabular}


La segunda variable ha de ser el aprovisionamiento de contenido, su propia difusión, el grado de presencia o extensión, así como del impacto con un marcado carácter macrocontextual, es decir, clara ruptura de las fronteras locales y nacionales para situarse en el plano de la internacionalidad.

Sin embargo, esta extensión internacional no se define solo por la vocación y motivación editorial de la revista, aun cuando una hipotética disponibilidad económica le permitiera articular una presencial real de esta naturaleza, siendo entonces otras las variables que han de ser consideradas a la hora de adquirir el estatus de universalidad, más próximas al concepto de calidad.

Esa internacionalización entonces vendrá dada y promovida por la capacidad de influencia científica que la revista llegue a generar, a partir de la calidad de sus contenidos, el interés de los científicos por los mismos, el impacto en términos de utilización y citaciones, etc., que acompañados de la amplitud de su difusión impregnan a ésta de ese carácter internacional (HERNÁNDEZ HUERTA, 2016).

No obstante, el grado de internacionalización de las revistas está normalmente acompañado de otras variables o recursos, a los que no siempre tienen acceso todos los editores de revistas. Así, el uso del inglés en la publicación de los artículos, el respaldo institucional, de grupos editoriales, el sometimiento a normas sólidas y estrictas de publicación o el apoyo de asociaciones científicas de reconocido prestigio pueden ser elementos determinantes a la hora de imprimir este carácter de internacionalidad a la revista.

Por tanto, reclutamiento de artículos, grado de indexación, índice de impacto, ámbitos de difusión, regularidad, rapidez de publicación, calidad y pertinencia son elementos determinantes en la oportunidad de tal internacionalidad.

\section{LA INTERNACIONALIZACIÓN COMO SINÓNIMO DE CALIDAD}

Inicialmente, entendemos que la internacionalización de la revista, por sí misma, ya debiera considerarse como un indicador de calidad, porque ampliar el circulo de interés de una revista científica ya le añade un valor superior que si solamente fuera local o de ámbito más restringido, y además porque alcanzar la amplitud del interés internacional es resultado del efecto multiplicador que deriva la "influencia esforzada" de la propia revista hasta lograr superar las fronteras locales y nacionales a las que, originariamente, se debe.

Pero, además, esto que, en otros momentos, o con la suficiente disponibilidad de recursos, pudiera ser más factible, en la actualidad, en un contexto altamente globalizado, con grandes dosis de competitividad y concurrencia de intereses tanto corporativos como 
económicos dificulta esa universalización si no se acompaña de otras variables que inciden directamente en la naturaleza del producto, definido con carácter universal como calidad.

Es decir, la universalización no es causa de..., sino la consecuencia lógica y necesaria de haber incorporado y superado unos indicadores de calidad mínima que hacen posible la universalización de la revista (REDVET, 2008).

Entre los indicadores más importantes de la calidad de las revistas que les facilita o sitúa en el contexto de la internacionalización están (DELGADO, RUÍZ-PÉREZ; JIMÉNEZCONTRERAS, 2016):

- Disponer de equipo editorial altamente profesionalizado

- Contar con un Comité científico cuyos miembros acumulen mérito y prestigio científico

- Contar con normas sólidas sobre el contenido, redacción y presentación de los artículos.

- Disponer de un sistema revisión y aceptación de contenidos formado por pares ciegos

- La pertinencia y solidez de los contenidos que se publican en términos de originalidad, novedad, interés, actualidad, rigor metodológico, relevancia, etc.

- Procedencia geográfica de los trabajos

- La frecuencia y regularidad en la publicación de los números de la revisita

- Capacidad de divulgación y difusión de los números publicados

- Indexación, que da cuenta de las oportunidades de extensión de la revista

- Impacto de la revista, que da cuenta del número de citas que recibe

No obstante, lo anterior, tal y como se señaló más arriba también hay otros indicadores de calidad con relación a la internacionalización de acuerdo a normas que los diversos ámbitos científicos han ido incorporando como es la utilización de la lengua inglesa para su comunicación (ARÉCHAGA, 2009), el respaldo de editores y asociaciones de investigadores.

Al respecto Ganga, Paredes y Pedraja-Rejas (2015) mencionan que: Las revistas académicas que han logrado mucha reputación y ascendencia son justamente aquellas que han conseguido ser reconocidas por índices definidos por entes constituidos por investigadores que respaldan la excelencia de estas, aceptando su incorporación a determinadas bases de datos (p1).

En consecuencia, se puede decir que la internacionalización de una revista es un potente indicador de calidad, no solo porque la internacionalización otorgue la calidad, sino porque alcanzar la internacionalización exige haber superado numerosos indicadores de tal calidad. 


\section{El Interés de Perspectiva Educacional de Promoverse en el Ámbito Internacional}

La Revista Perspectiva Educacional es una publicación bi-anual, con una trayectoria de 35 años en el ámbito de la Educación. Nace en la Escuela de Pedagogía de la Facultad de Filosofía y Educación de la Pontificia Universidad Católica de Valparaíso, con la finalidad de comunicar los aportes de académicos nacionales y extranjeros en la construcción y desarrollo del conocimiento profesional en educación. En la década del ' 90 se constituye en una de las revistas de educación con mayor presencia ininterrumpida a nivel nacional.

En términos de internacionalización, a partir del año 2010 Perspectiva Educacional inicia un nuevo proceso de edición, actualizando su formato de edición impreso a un formato digital a través de la plataforma Open Journal System (OJS), que permite otorgar mayor visibilidad y cobertura a nivel mundial, con una política de libre acceso, bajo el principio de poner a disposición del público de manera gratuita la investigación y reflexión teórica, favoreciendo de esta manera el intercambio del conocimiento.

Perspectiva Educacional se renueva buscando responder a los criterios de calidad internacionales anteriormente mencionados por Delgado, Ruíz-Pérez y Jiménez-contreras, 2016 que son a la vez las directrices que rigen las colecciones SciELO. Entre los indicadores más importantes de calidad a los que responde Perspectiva Educacional se encuentran:

a) Disponer de equipo editorial altamente profesionalizado

La literatura indica que contar con un equipo editorial altamente especializado es un importante indicador para definir la calidad de una revista. Al respecto, Delgado LópezCózar, Ruiz-Pérez y Jiménez-Contreras (2006) señalan que: La existencia de comités editoriales, encargados de asegurar el control de calidad de los contenidos, y la Edición de Revistas Científicas: Directrices, Criterios y Modelos de Evaluación compuestos por reputados científicos con amplio currículo como investigadores en activo y experiencia editorial, es fundamental para el buen desenvolvimiento de la revista (p.63)

En este sentido la revista Perspectiva Educacional cuenta con un consejo editorial altamente especializado conformado por 12 especialistas en el ámbito de la educación de reconocida trayectoria. Un $66 \%$ del equipo corresponde a integrantes internacionales provenientes de 7 países y un $8 \%$ del Comité editorial corresponden a investigadores de Chile. (Ver Gráfico 1) 


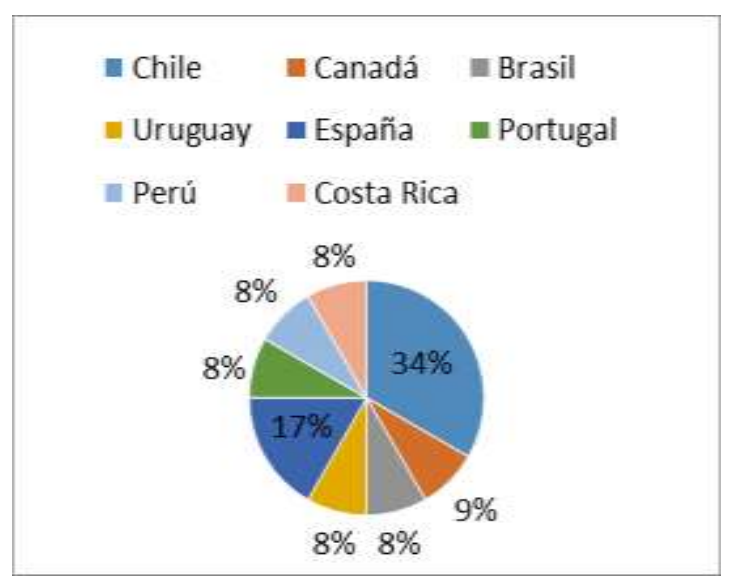

Gráfico 1: Consejo Editorial

Fuente: Las autoras.

b) Contar con un Comité científico cuyos miembros acumulen mérito y prestigio científico. Según estudios realizados por Delgado López-Cózar, Ruiz-Pérez y JiménezContreras (2006) en términos de internacionalización contar con un comité científico con diversa procedencia geográfica otorga a la revista la oportunidad de "atraer hacia ella a científicos reputados y de abrirla al exterior, con lo que se puede asegurar una mayor objetividad y neutralidad, un mayor acierto en el diseño de la política editorial y una mayor proyección de la revista" (p.64).

Dentro de este marco Perspectiva Educacional dispone de un comité científico especializado con representantes en 12 países, de los cuales solo un $14 \%$ pertenece a investigadores chilenos. (Ver Gráfico 2)

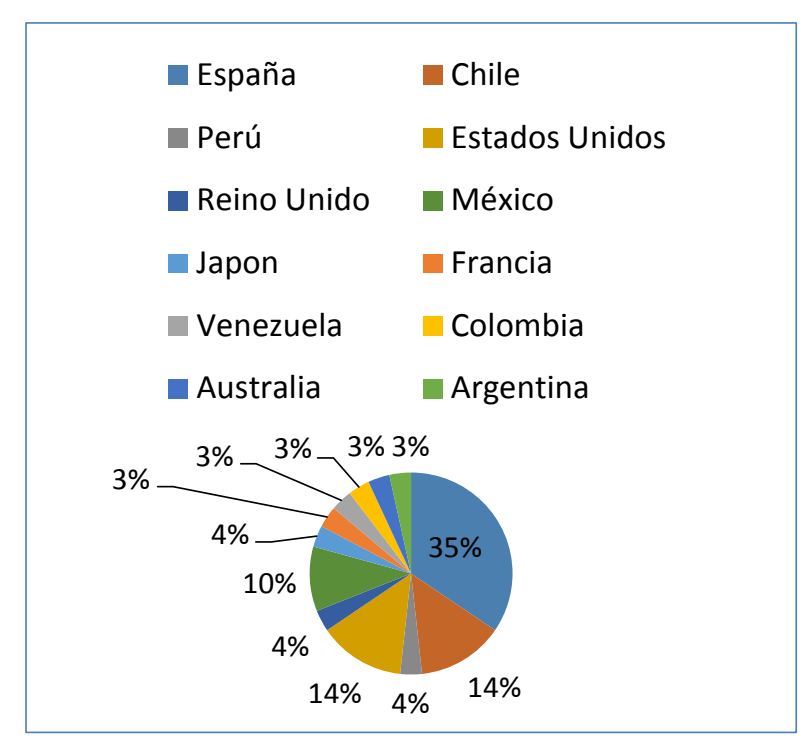

Gráfico 2: Comité Científico

Fuente: Las autoras. 
c) Normas sólidas sobre el contenido, redacción y presentación de los artículos

Las normas internacionales de presentación de publicaciones periódicas son un elemento importante de considerar, pues tal como lo señalan (DELGADO LÓPEZ-CÓZAR, 1999; DELGADO LÓPEZ-CÓZAR, RUIZ PÉREZ ; JIMÉNEZ-CONTRERAS, 1999 citado en RUIZ-PÉREZ, DELGADO LÓPEZ-CÓZAR; JIMÉNEZ-CONTRERAS, 2006) "La importancia atribuida a este apartado se justifica por la trascendencia que tiene una revista como instrumento de comunicación, difusión e impacto de sus contenidos científicos (p. 407)."

Es por ello que Perspectiva Educacional establece normas sólidas sobre el contenido de los artículos que publica, velando por su relevancia, originalidad y contribución a la mejora de la calidad de los sistemas educativos. En este sentido Perspectiva Educacional declara en su normativa para autores la estructura que deben seguir los artículos que son enviados a la revista y tipo de artículos que recibe; artículos de investigación científica y artículos teórico-reflexivos.

En cuanto a su redacción, Perspectiva Educacional busca responder a los criterios establecidos por SciELO donde se indica que los artículos deben poseer una descripción estructurada, que explique la investigación realizada, manejando un lenguaje claro y una redacción óptima. Para velar con este indicador los artículos son sometidos a una primera fase de revisión con el editor, donde se cautela que los originales cumplan con las políticas de pertinencia temática, novedad y normas editoriales. Al respecto se detalla el proceso de edición que siguen los artículos descrito en la plataforma virtual de la revista. (Perspectiva Educacional, 2016): La revista recibe originales correspondientes a trabajos teóricos o empíricos en el ámbito de la formación de profesores. Entre los tipos de trabajos se incluyen: artículos de investigación científica y artículos teórico-reflexivos.

Los artículos son sometidos a un riguroso proceso que considera las siguientes fases:

- Recepción del manuscrito.

- Revisión inicial del equipo Editor.

- Evaluación ciega de pares externos.

- Segunda versión del manuscrito.

- Segunda ronda de revisión de pares externos.

- Dictamen final.

- Publicación 


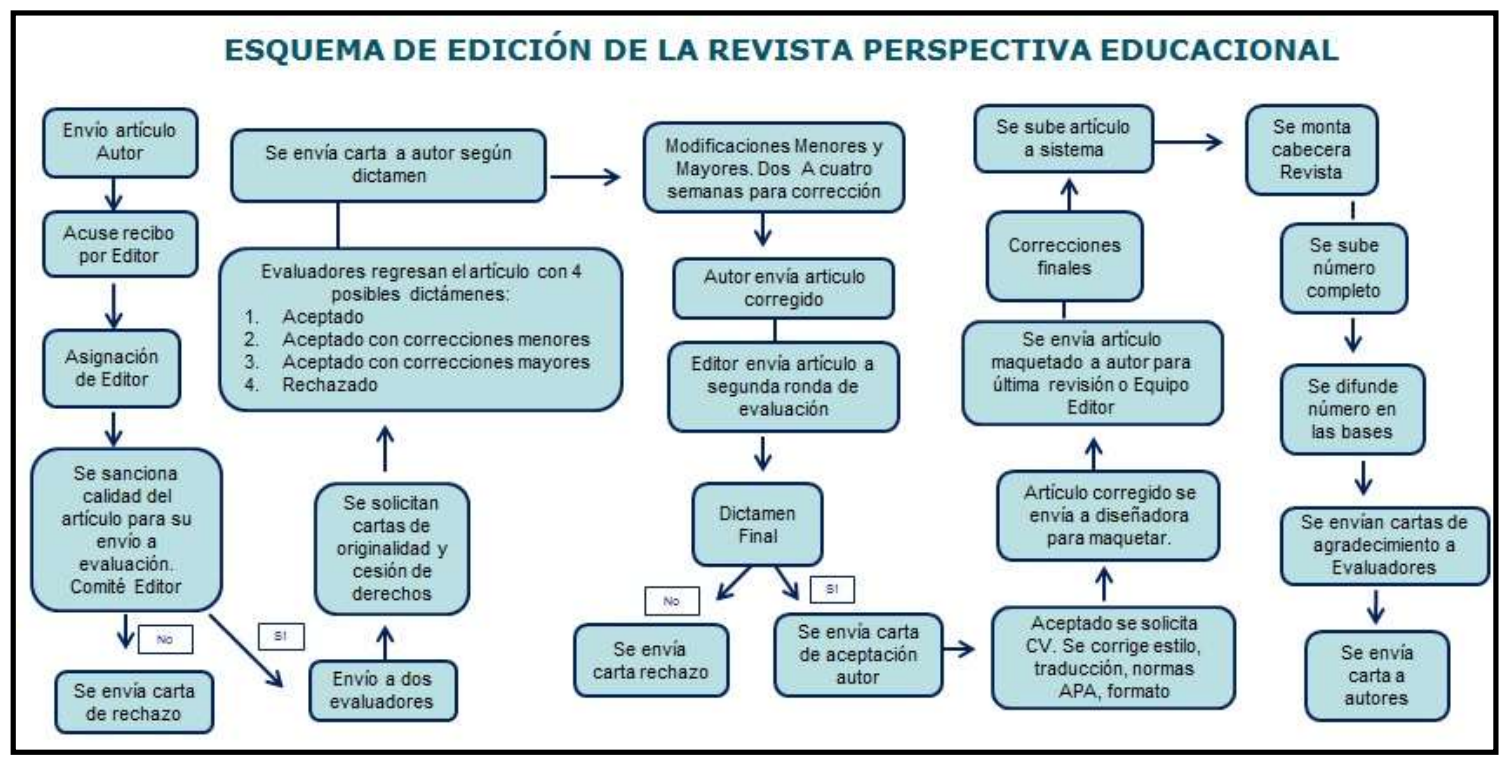

Figura 1: Esquema de edición de la Revista Perspectiva Educacional Fuente:

http://www.perspectivaeducacional.cl/index.php/peducacional/pages/view/Proceso\%20de\%20Edición

d) Sistema revisión y aceptación de contenidos formado por pares ciegos

Perspectiva Educacional mantiene una especial preocupación por el proceso de revisión de sus artículos y los somete a una rigurosa evaluación por pares, que demora entre uno y seis meses.

Cuando el equipo editor decide que el artículo puede ser enviado a evaluación externa, se asignan dos revisores ciegos, quienes, a través de la plataforma de la revista completan la pauta de evaluación respectiva, ya sea para un artículo teórico o de investigación.

La evaluación concluye con un dictamen que indica si un manuscrito es: Aceptado, aceptado con modificaciones menores, aceptado con modificaciones mayores, o rechazado. En el formato de evaluación se solicita a los pares: a) indicar el cumplimiento o no de criterios de forma y contenido, b) Realizar una valoración general del artículo incluyendo sugerencias y/o aportes.

En caso de existir discrepancias entre los dictámenes de los evaluadores, se envía el manuscrito a un tercer evaluador y con este tercer dictamen, el comité editor toma la decisión de rechazar o enviar el artículo para su modificación.

Los evaluadores corresponden a académicos expertos en el tema, externos al comité editor y, preferentemente, de países de ubicación geográfica distinta a la de los autores del artículo. 


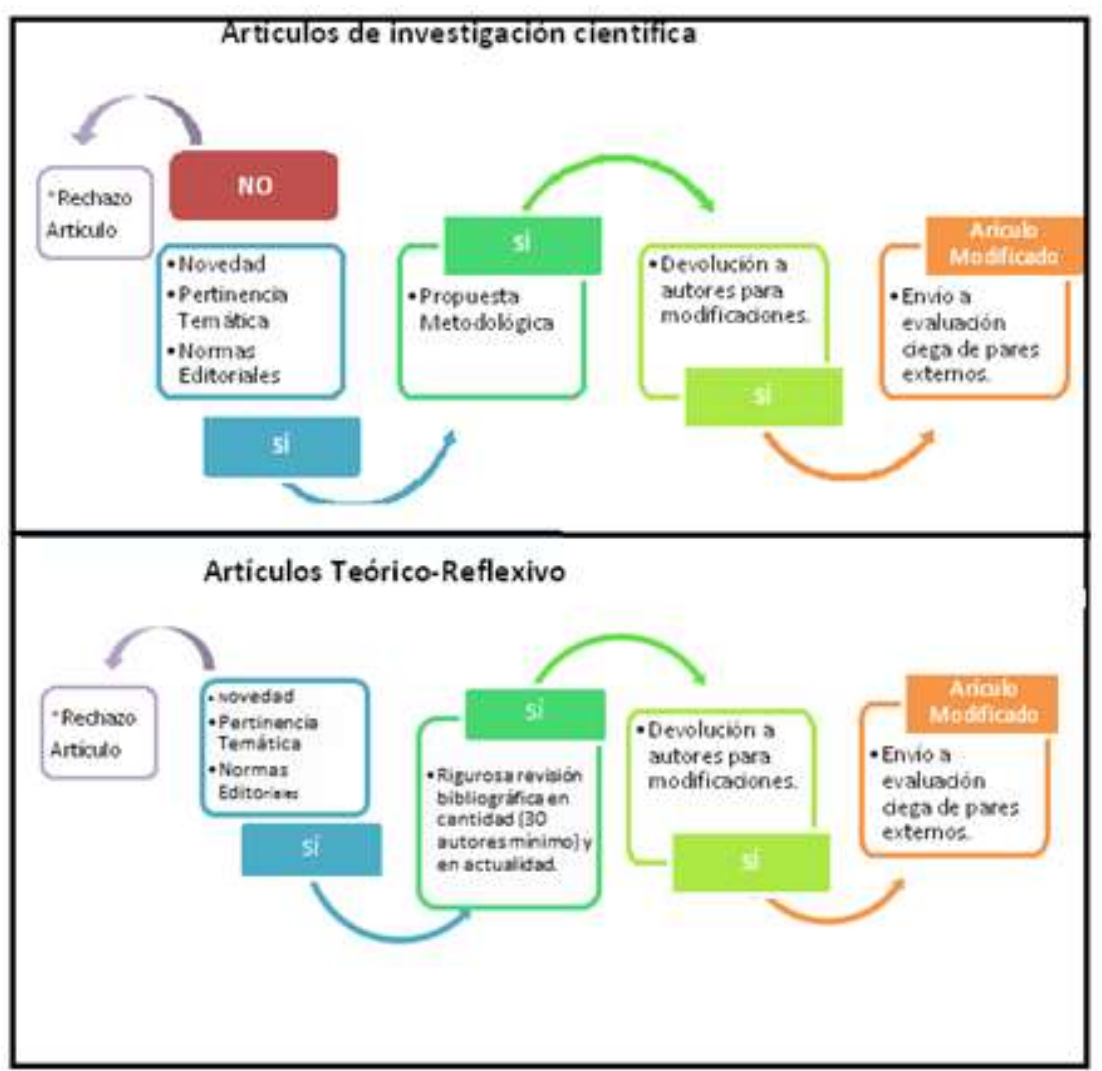

Figura 2: Esquema de ciclo de revisión inicial para artículos. Fuente:

http://www.perspectivaeducacional.cl/index.php/peducacional/pages/view/Proceso\%20de\%20Edici\%C3\%B3n

e) Procedencia geográfica de los autores.

En términos de procedencia geográfica de los trabajos publicados, Perspectiva Educacional ha aumentado su flujo de artículos.

Entre los años 2010 y 2011 Perspectiva Educacional publicó 15 artículos provenientes de los países; España Chile México y Canadá, comprendiendo durante este periodo sólo los 4 países mencionados. Mientras que en los años 2015 y 2016 se publicaron 40 artículos abarcando 10 países, aumentando a un $250 \%$ su cobertura. 


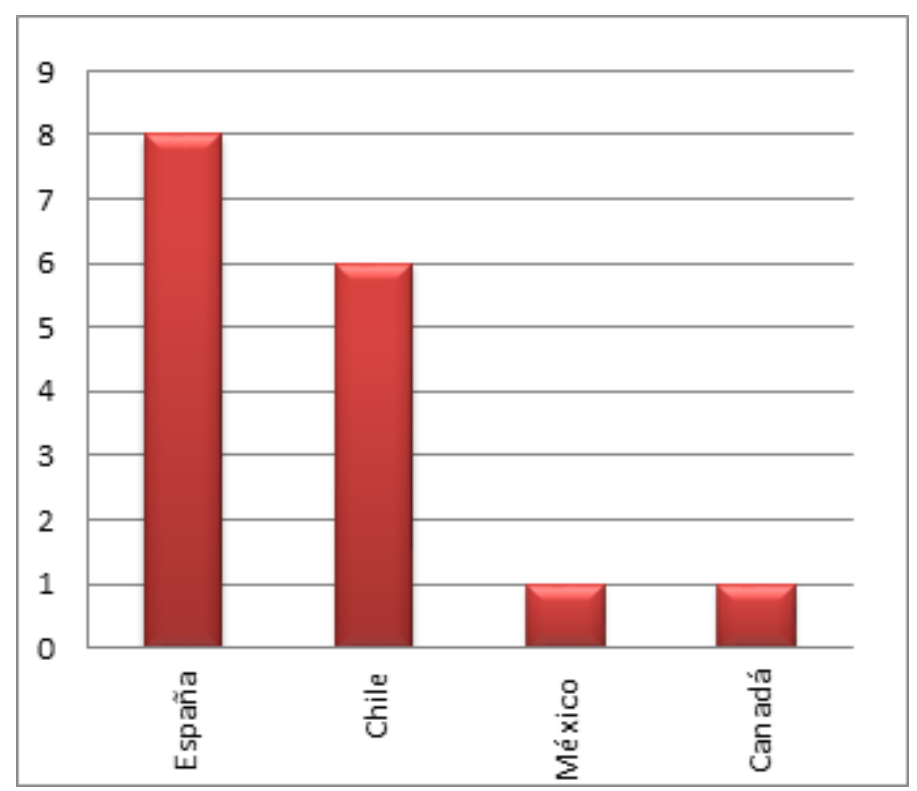

Gráfico 3: Procedencia geográfica de los autores (2010-2011)

Fuente: las autoras.

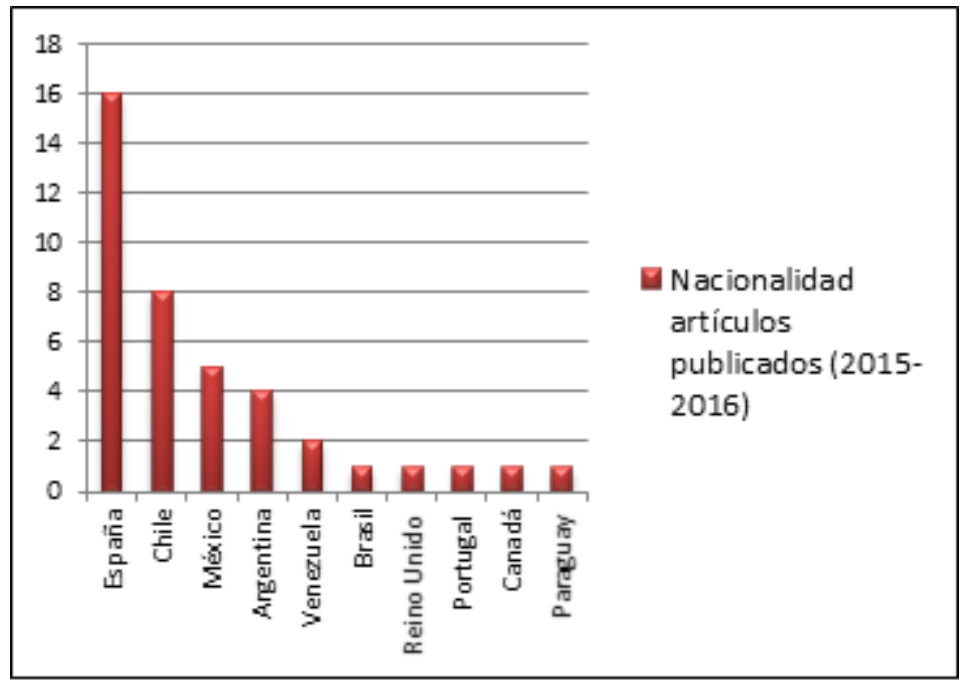

Gráfico 4: Procedencia geográfica de artículos publicados entre los años 2015-2016

Fuente: las autoras.

\section{Posicionamiento Iberoamericano de Perspectiva Educacional}

La Revista Perspectiva Educacional pose una trayectoria de más de 30 años de publicación en el ámbito de la Educación. El primer número se publica en el año 1980 con la finalidad de comunicar los aportes de académicos nacionales y extranjeros que participan en los Seminarios, Congresos y actividades organizadas por el Instituto de Educación, especialmente en las áreas de Teoría de la Educación, Historia de la Educación, Currículum, Evaluación, Nuevas Tecnologías de la Información y Comunicación (TICS) y estudios comparados, como también los productos de las investigaciones desarrolladas en los 
departamentos de la Escuela de Pedagogía e Instituto de Educación.

En la década del 90' se constituye en una de las revistas de educación de mayor permanencia ininterrumpida a nivel nacional. En el año 2007 se suspende su publicación periódica a la luz de nuevas demandas y políticas institucionales que privilegian las ediciones digitales.

A partir del año 2010, la revista Perspectiva Educacional se encuentra en una fase de renovación que especifica una línea de especialización de publicación científica en el ámbito de la formación inicial y continúa del profesorado. Actualiza su formato de edición impreso a online a través de la plataforma Open Journal System (OJS) de acuerdo a los requerimientos de los nuevos tiempos. Se constituye como la única revista científica en Chile específica en la formación inicial y continua de profesores, existiendo en el país solo una revista del ámbito educacional con indexación SciELO (Estudios Pedagógicos) y otras publicaciones de carácter profesional, informativas y de apoyo al docente.

En la actualidad Perspectiva Educacional busca convertirse en un referente en la formación inicial y continua de profesores, así como también alcanzar la internacionalización. Lo anterior lleva a la revista a buscar acciones que incrementen su visibilidad y posicionamiento en el mundo académico.

\section{Aumento en el Flujo de Artículos}

El gráfico da cuenta del flujo de artículos de Perspectiva Educacional, entre los años 2010 al 2016. En julio del año 2013, Perspectiva Educacional logra su indización en Redalyc, a partir de este hito se logra un notable incremento de un $72 \%$ en el flujo de artículos recibidos, aumento que se mantiene en el tiempo, sin embargo es en el año 2016 cuando Perspectiva Educacional alcanza mayor visibilidad a nivel internacional con su indización en la base Emerging Source Citation Index (ESCI) de Thompson Reuters apreciándose un importante aumento en su flujo de artículos con respecto a años anteriores.

Actualmente la revista se encuentra indizada en: Emerging Source Citation Index (ESCI) de Thompson Reuters, Latindex, Dialnet, Ebsco, Iresie, DOAJ, Redalyc, E-revistas, CEsic, Redib, posicionándose como una revista emergente. 


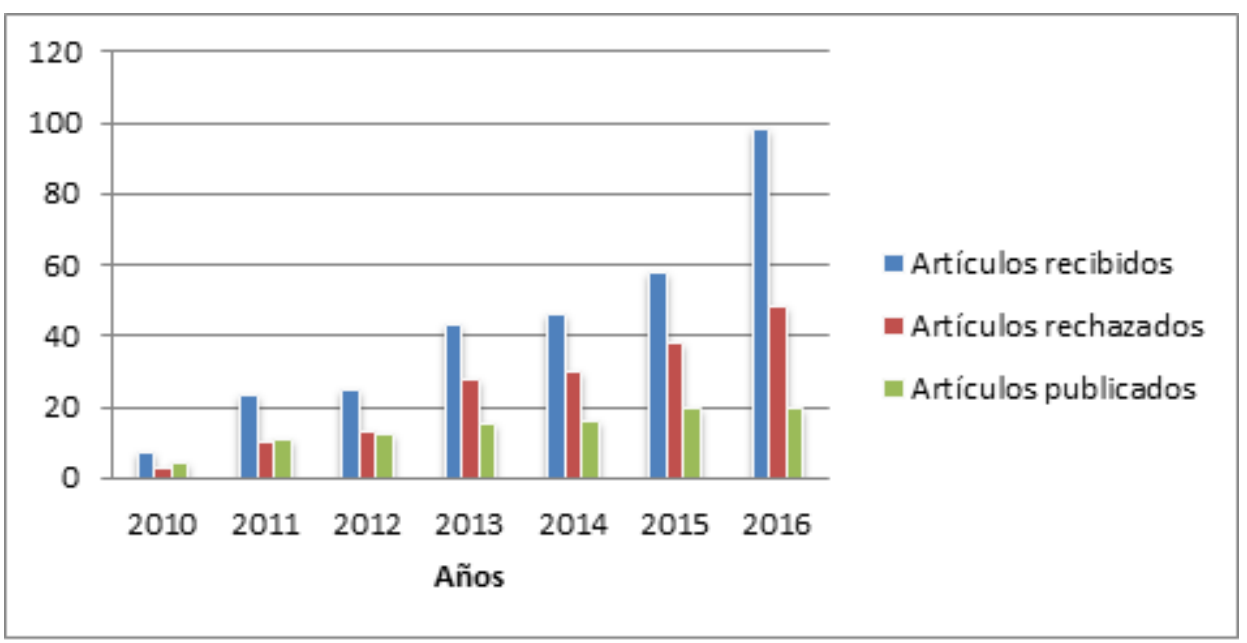

Gráfico 5: Flujo de artículos Perspectiva Educacional (2011-2016)

Fuente: http://www.perspectivaeducacional.cl/index.php/peducacional/index

\section{Incremento de Lectores de Perspectiva Educacional}

El posicionamiento a nivel Iberoamericano de Perspectiva Educacional ha contribuido al aumento de visitas al sitio de la revista. Los datos obtenidos de google analytics, dan cuenta de que Perspectiva Educacional ha logrado un notable incremento en el número de visitas recibida es así como entre los años 2011-2012, la revista alcanzó 3.510 visitas registradas por usuarios provenientes de Chile mientras que, en el año 2016, la revista llegó a obtener 23.950 visitas, registrando a México como el país que más usuarios consigue.

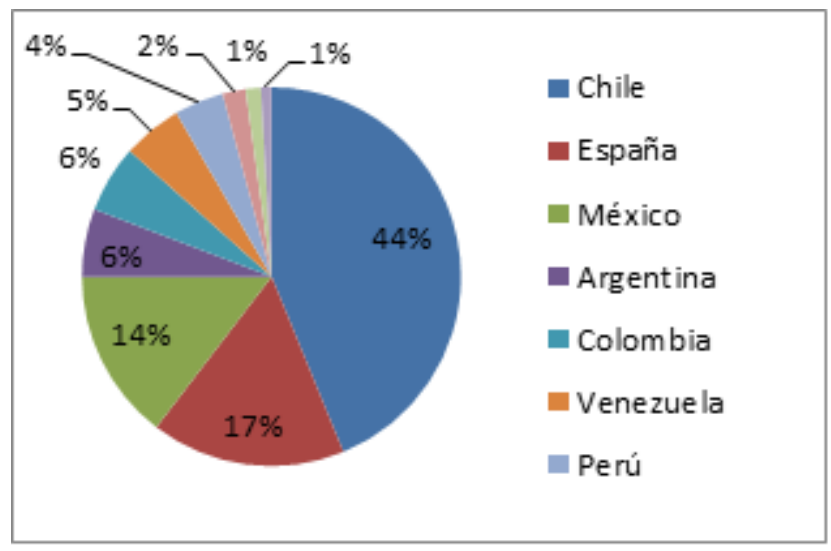

Gráfico 6: Visitas Revistas 2011 -2012

Fuente: Las autoras.

\section{Acciones de Impulso}

De acuerdo con Díaz y Ortiz (2014) Para conseguir la difusión y el impacto en la audiencia de los contenidos de las revistas digitales es necesario emprender acciones que se pueden denominar de impulso y también acciones de atracción (p.8). 
Las acciones de impulso tienen como objetivo hacer llegar de manera interactiva la revista y sus contenidos a destinatarios concretos (DÍAZ; ORTÍZ, 2014). Cumpliendo con este objetivo, Perspectiva Educacional genera distintas bases de dato con la finalidad de aumentar el número de lectores y atraer a personas especialistas en el ámbito de la Educación interesadas en comunicar sus aportes académicos producto de revisiones teóricas 0 investigaciones científicas en el área.

La revista también busca generar convenios de colaboración con instituciones de educación superior para fortalecer la difusión de los artículos publicados y establecer cooperación académica con otras revistas.

\section{Revistas de Intercambio}

La revista cuenta con "Revistas de intercambio" con la finalidad de desarrollar acciones que promuevan la colaboración académica con otras revistas a nivel nacional e internacional para contribuir a la difusión de los artículos y enriquecer la producción científica en Educación. La tabla muestra las revistas con las que Perspectiva Educacional mantiene relación. (Ver tabla 1).

Tabla 1: Colaboración académica con Revistas de intercambio

\begin{tabular}{|l|l|}
\hline Revista Académica & País \\
\hline $\begin{array}{l}\text { Revista Pensamiento Educativo } \\
\text { Latinoamericano }\end{array}$ & Chile \\
\hline Revista Padeia & Chile \\
\hline Revista Estudios Pedagógicos & Chile \\
\hline Revista Ciencias Religiosas & Chile \\
\hline Revista Temas Sociológicos & Chile \\
\hline Revista AKADEMEIA & Chile \\
\hline Revista Comunicar & España \\
\hline Revista educación XX1 & España \\
\hline $\begin{array}{l}\text { Revista Iberoamérica de Educación a } \\
\text { Distancia }\end{array}$ & España \\
\hline Revista EARI & España \\
\hline Revista Educar & España \\
\hline
\end{tabular}




\begin{tabular}{|l|l|}
\hline Revista Perfiles Educativos & México \\
\hline Revista Aula Abierta & México \\
\hline Revista REIEC & Argentina \\
\hline
\end{tabular}

Fuente: Elaboración propia.

\section{Boletín Educativo}

A partir del año 2014, La Revista Perspectiva Educacional inicia acciones de difusión a través de la edición de un Boletín Educativo a fin de que sus artículos publicados sean consultados por distintos actores del ámbito de la educación escolar, como por ejemplo directores, profesores, y otros profesionales que conforman la comunidad de los establecimientos educacionales chilenos. En este sentido el boletín de la revista surge como un aporte al sistema escolar, buscando nutrir a la comunidad escolar con conocimiento generados por la investigación educativa. (LEIVA et al., 2016).

En el mes de octubre del año 2014 se edita el primer Boletín Educativo para docentes y directivos de los establecimientos escolares de la Región de Valparaíso, invitando a la lectura y discusión académica y al desarrollo profesional docente a los actores clave del sistema escolar: profesores y directivos (LEIVA et al., 2016).

Los resultados de esta experiencia fueron positivos y agradecidos por la comunidad escolar. Al respecto se puede afirmar que las escuelas agradecieron el envío de forma impresa y virtual del boletín y cinco escuelas municipales señalaron el aporte del material al desarrollo de la educación en las escuelas y liceos más vulnerables de la Región de Valparaíso (LEIVA et al., 2016, p.13).

\section{Conclusiones}

Las revistas científicas son un instrumento eficaz para la internacionalización, pues responden a una idea básica del quehacer científico, como es la comunicación y el intercambio de ideas, conocimiento y hallazgos de investigaciones de académicos, investigadores, profesores y profesionales de la educación de distintos lugares del mundo, con sus sellos, tradiciones culturales y académicas que enriquecen la realidad educativa al mirarla desde distintos prismas y enfoques.

Al respecto, las tecnologías de la información y comunicación han contribuido significativamente en este aspecto, al brindar un soporte tecnológico que permite alojar revistas para acceder a una mayor visibilidad y difusión a nivel internacional.

En relación a la revista Perspectiva Educacional las acciones mencionadas dan cuenta de una prolífica actividad en el ámbito editorial y el compromiso por difundir la investigación y construcción de conocimiento en Educación.

\begin{tabular}{l|l|l|l|l|l|l}
\hline (C) Rev. Inter. Educ. Sup. & Campinas, SP & v.2 & n.3 & p.480-500 & set./dez. 2016 & ISSN 2446-9424 \\
\hline
\end{tabular}


Por ello, su misión es continuar posicionando a Perspectiva Educacional como una de las mejores revistas del ámbito educativo, específicamente sobre la formación inicial y continua de profesores de América Latina, manteniendo su calidad académica y potenciando su visibilidad e internacionalización.

\section{Poyecciones y Reflexiones Finales}

Uno de los principales desafíos de Perspectiva Educacional es aumentar su visibilidad a nivel nacional, latinoamericano y mundial. Tal como lo indican (SANTORO et al., 2013) una de las maneras para medir la visualización internacional es a través de su índice de impacto, el que fue definido por Thomson Reuters como el número de referencias promedio que recibe una revista en un año determinado de los 'ítems citables' publicados en esa revista durante los dos años previos (DORTA-GONZÁLEZ; DORTA-GONZÁLEZ, 2014, p.1).

De lo anterior se desprende que es fundamental para una revista que desea alcanzar su internacionalización publicar artículos resultantes de proyectos de investigación tanto en el idioma español como en inglés y de esta manera aumentar el número de citas promedio recibidas, por lo que uno de los principales objetivos de Perspectiva Educacional es atraer a autores de alto nivel en el ámbito del conocimiento a nivel internacional y de llegar a ser conocida en distintos países en donde la revista presenta un menor número de visitantes y lectores. Una revista que publica artículos de calidad recibirá muchas citas y tendrá un elevado Factor de Impacto (GÁMEZ, 2013).

Las imágenes extraídas de google analitycs, dan cuenta de cómo Perspectiva Educacional ha ido incrementando su cobertura internacional en países de habla hispana.

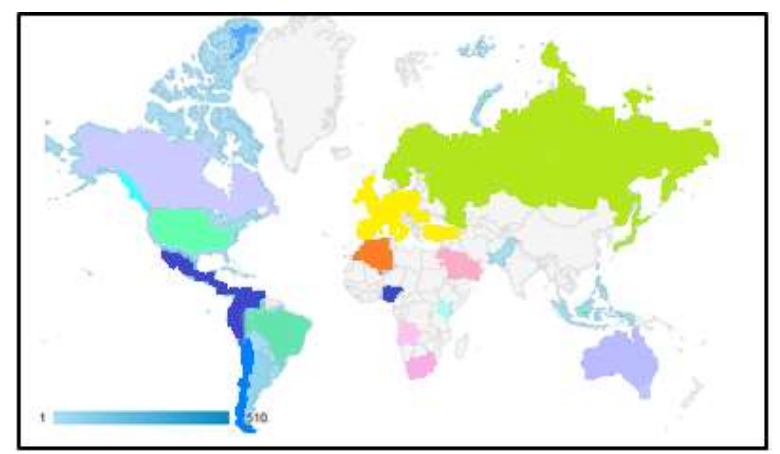

Figura 3: Visitas Revista año 2011-2012

Fuente: Perspectiva Educacional (2011-2012). Recuperado de:

file:///C:/Users/Natalia/Downloads/Analytics\%20www.perspectivaeducacional.cl\%20Ubicaci\%C3\%B3n\%2020 110504-20120504.pdf 


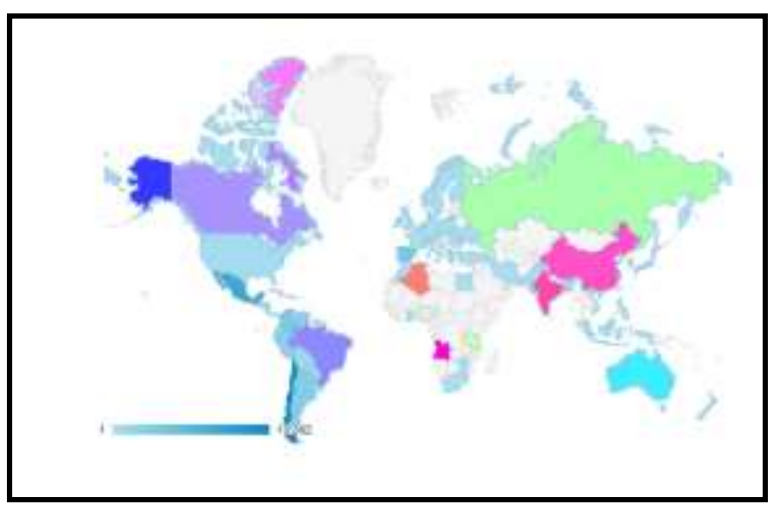

Figura 4: Visitas Revista año 2012-2013

Fuente: Perspectiva Educacional (2012-2013). Recuperado de:

file://C:/Users/Natalia/Downloads/Analytics\%20www.perspectivaeducacional.cl\%20Ubicaci\%C3\%B3n\%2020 110504-20120504.pdf

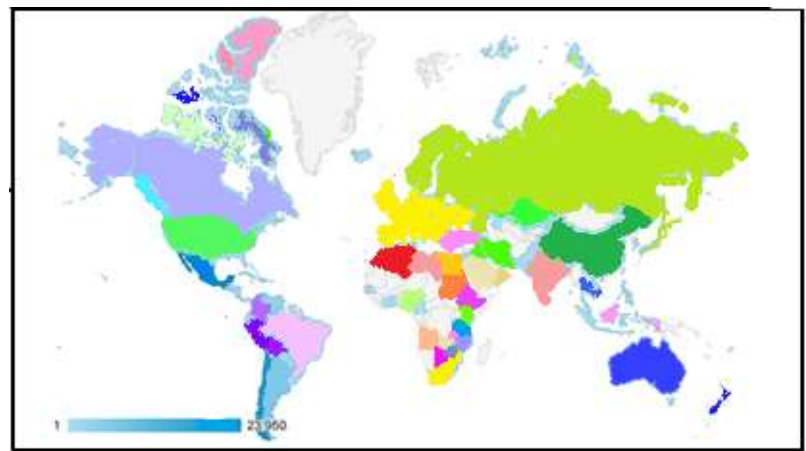

Figura 5: Visitas Revista año 2014-2015

Fuente: Usuarios registrados en Perspectiva Educacional (2016). Recuperado de:

file://C:/Users/Natalia/Downloads/Analytics\%20www.perspectivaeducacional.cl\%20Ubicaci\%C3\%B3n\%2020 160101-20160922\%20(1).pdf

Por otra parte, de los datos analizados también se puede interpretar que la cobertura y posicionamiento de la revista tiene relación con las bases de dato en las que se encuentra indizada. Es así como en los años 2013 y 2016, la revista sufre un importante incremento en el número de visitas y artículos recibidos debido a su indización en Redalyc (2013) y (ESCI) de Thompson Reuters (2016).

Actualmente la revista trabaja para posicionarse como un referente internacional en el ámbito académico buscando cumplir con los estándares de indización de reconocidas bases de dato tales como; Scopus Thomson Reuters (ISI), ya que tal como lo señala López Leyva (2011, p. 1): "La literatura consultada sobre la visibilidad de las publicaciones supone que el ingreso de las revistas científicas a los bancos de datos de mayor prestigio, constituye un camino para que éstas sean consultadas por todos los usuarios en el mundo sin ninguna barrera".

Por otra parte, para los investigadores, la decisión de donde publicar los hallazgos de sus estudios, también está determinada por la indización de la revista, pues el prestigio de esta depende del mismo (GALÁN, 2015). 
A partir de lo expuesto, queda claro que las revistas científicas indizadas en las bases de alto impacto (Scopus, Thomson Reuters (ISI) pueden lograr una mayor internacionalización, pero es necesario preguntarse, a nivel latinoamericano ¿Cuántas Revistas de educación están indizadas en Thomson Reuters (ISI) catálogo principal? ¿Cómo las revistas Latinoamericanas logran mayor internacionalización, si pese a todas las iniciativas y esfuerzos que realizan, Thomson Reuters no ingresa revistas latinoamericanas a su colección? ¿Cómo se logra la equidad editorial en revistas latinoamericanas que no poseen los recursos y condiciones editoriales? ¿Cómo se revitaliza a revistas latinoamericanas que lo están haciendo bien, tienen gran flujo de artículos y lectura de sus ediciones de una amplia cobertura geográfica, para mantenerse esperanzadas de lograr estas indizaciones y no morir en el intento?

Perspectiva Educacional se reinventa y proyecta acciones año a año para lograr la plena internacionalización, el problema está en que es una competencia poco justa, con desigualdad de condiciones, entre equipos editoriales que llevan mucho más tiempo, han inventado ellos las reglas y algunas de ellas les favorecen claramente (idioma inglés) (Galán, 2015), en fin, esperemos sobrevivir, pues prueba de buen trabajo hemos dado.

\section{Referências}

ALTBACH, P., REISBERG, L., RUMBLEY, L. Trends in global higher education, tracking an academic revolution. París: UNESCO, 2009.

ARÉCHAGA, J. Internacionalización de las revistas científicas españolas: nuestra asignatura pendiente. ELSEVIER, v.4, n.10, 2009.

CALOGERO M. SANTORO, STANDEN, VIVIEN G, Dante, ANGELO; GAVILÁN, VIVIAN. Internacionalización de Revistas Científicas en Campos Emergentes como Antropología: Desafíos y Oportunidades para Chungara. Chungará, Arica, v.45, n.3, p.367369, 2013. Disponível em: <https://dx.doi.org/10.4067/S0717-73562013000300001>. Acesso em: 20 oct. 2016.

DE WIT, H. Globalización e internacionalización de la educación superior. Revista de Universidad y Sociedad del Conocimiento, v.8, n. 2, p.77-84, 2011.

DELGADO, E.; RUIZ PÉREZ, R. Y JIMÉNEZ-CONTRERAS, E. La edición de revistas científicas, directrices, criterios y modelos de evaluación. Granada: Universidad de Granada, 2016.

DÍAZ, M. Y ORTIZ, Z. Indicadores de calidad para la visibilidad de las revistas científicas electrónicas de la Universidad del Zulia. Enlace: Revista Venezolana de Información, Tecnología y Conocimiento, v.11, n. 2, p.91-111, 2014.

GALÁN, A. ¿Editores o "pringaos”? Jugando la liga del factor de impacto. Aula Magna 2.0. [Blog, 18 de diciembre de 2015].

\begin{tabular}{l|l|l|l|l|l|l}
\hline (C) Rev. Inter. Educ. Sup. & Campinas, SP & v.2 & n.3 & p.480-500 & set./dez. 2016 & ISSN 2446-9424 \\
\hline
\end{tabular}


GANGA, F. PAREDES, L; PEDRAJA-REJAS, L. Importancia de las publicaciones académicas: algunos problemas y recomendaciones a tener en cuenta. Idesia, Arica, v.33, n.4, p.111-119, 2015.

GÁMEZ, MATÍAS. La evaluación de revistas de psicología: correlación entre el factor de impacto, el índice h y los criterios de Latindex. Investigación bibliotecológica, v.27, n.61, p.15-27, 2013. Disponível em: <http://www.scielo.org.mx/scielo.php?script=sci_arttext \&pid=S0187-358X2013000300002\&lng=es\&tlng=es >. Acesso em: 20 oct. 2016.

HERNÁNDEZ, H. La gestión editorial de revistas científicas hoy. La internacionalización de la comunicación científica. Aula Magna 2.0. [Blog, 22 de enero de 2016], en http://cuedespyd.hypotheses.org/1072.

KNIGHT, J. Higher education in turmoil: the changing world of internationalization. Rotterdam: Sense Publishers, 2008.

KNIGHT, J.. Internacionalización de la Educación Superior: Nuevos desarrollos y Consecuencias no Intencionadas. UNESCO - Iesalc Informa de Educación superior, n.211, 2010.

LEIVA, MV., MUÑOZ, C., CONEJEROS, L, GOLDRINE, T. Boletín Educativo: una experiencia de acercamiento al género académico para docentes de escuelas chilenas. Revista Mexicana de Investigación Educativa, v.71, n.21, 2016. Disponível em: <http://www. comie.org.mx/v1/revista/portal.php?idm=es\&sec=SC03\&sub=SBB\&criterio=ART71011 > . Acesso em: 20 oct. 2016

LÓPEZ LEYVA, S. Visibilidad del conocimiento mexicano. La participación de las publicaciones científicas mexicanas en el ámbito internacional. Revista de la educación superior, v.40, n.158, p.151-165, 2011. Disponível em: <http://www.scielo.org.mx/ scielo.php?script=sci_arttext\&pid=S0185-27602011000200010\&lng=es\&tlng=es $>$. Acesso em: 21 oct. 2016.

PALAMIDESSI, M., GOROSTIAGA, J., Y SUASNÁBAR, C. "El desarrollo de la investigación educativa y sus vinculaciones con el gobierno de la educación en América Latina”. Revista Perfiles Educativos XXXVI, México, v.143, p.49-66, 2014.

PACKER, A. La internacionalización de las revistas fue el tema central de la IV Reunión Anual de SciELO. Revista Scielo en Perspectiva, 2014. Disponível em:

$<$ http://blog.scielo.org/es/2014/12/16/la-internacionalizacion-de-las-revistas-fue-el-temacentral-de-la-iv-reunion-anual-de-scielo/\#.WCIP1oWcGUk>. Acesso em: 20 oct. 2016.

REDVET. Los 56 Criterios FECYT para la evaluación de la calidad de las revistas científicas españolas. Revista electrónica de Veterinaria, v.9, n. 9, 2008. Disponível em: <http://www. veterinaria.org/revistas/redvet/n090908.html>. Acesso em: 20 oct. 2016.

ZYCH, I.; BUELA-CASAL, G. Internacionalidad de las revistas de psicología multidisciplinar editadas en Iberoamérica. Universitas Psychologica, v.9, n.1, 27-34, 2010. 
RUIZ-PÉREZ, R., LÓPEZ-CÓZAR, DELGADO, E., JIMÉNEZ-CONTRERAS, E. Criterios del Institute for Scientific Information para la selección de revistas científicas. Su aplicación a las revistas españolas: metodología e indicadores International Journal of Clinical and Health Psychology, vl. 6, n. 2, mayo, p. 401-424, 2006.

DORTA GONZÁLEZ, M.I, \& DORTA GONZÁLEZ, P. Factor de impacto agregado según campos científicos. Investigación bibliotecológica, v.28, n.62, 15-28. Epub 00 de abril de 2014. Disponível em: <http://www.scielo.org.mx/scielo.php?script=sci_arttext\&pid=S0187358X2014000100002\&lng=es\&tlng=es $>$. Acesso em: 20 oct. 2016. 